\title{
Sex differences in health services and medication use among older adults with asthma
}

\author{
Teresa To ${ }^{1,2,3}$, Natasha Gray ${ }^{1}$, Kandace Ryckman ${ }^{1}$, Jingqin Zhu ${ }^{1,3}$, Ivy Fong (i) \\ and Andrea Gershon (10) $1,2,3,4$
}

Affiliations: ${ }^{1}$ Child Health Evaluative Sciences, The Hospital for Sick Children, Toronto, ON, Canada. ${ }^{2}$ Dalla Lana School of Public Health, University of Toronto, Toronto, ON, Canada. ${ }^{3}$ ICES, Toronto, ON, Canada. ${ }^{4}$ Sunnybrook Health Sciences Centre, Toronto, ON, Canada.

Correspondence: Teresa To, Child Health Evaluative Sciences, Peter Gilgan Centre for Research and Learning, The Hospital for Sick Children, 686 Bay St, Toronto, ON, M5G 0A4, Canada. E-mail: teresa.todsickkids.ca

ABSTRACT Sex differences are well documented in chronic disease populations with cardiovascular disease and diabetes. Although recent research has suggested that asthma is more severe in older women compared to men, the extent of this difference remains poorly understood. The objective of this study was to compare rates of asthma-specific health services use (HSU) and medication use, between older women and men with asthma.

This population-based cohort study included 209054 individuals aged $\geqslant 66$ years with asthma from health administrative data in Ontario, Canada. The primary exposure was sex. Outcomes included asthmaspecific HSU (spirometry, emergency department (ED), hospitalisation, physician office and specialist visits) and medication use (asthma controller and reliever prescriptions). Negative binomial regression models adjusted for age, socioeconomic status and comorbidities were used to ascertain outcomes by sex from 2010 to 2016.

Compared to men, women had lower rates of spirometry (adjusted relative rate (ARR) 0.87, 95\% CI 0.85-0.89) and specialist visits for asthma (ARR 0.93, 95\% CI 0.90-0.96), but higher rates of asthmaspecific ED (ARR 1.43, 95\% CI 1.33-1.53) and physician office visits (ARR 1.03, 95\% CI 1.01-1.05). Women also had lower asthma controller (ARR 0.98, 95\% CI 0.97-0.99) but higher asthma reliever (ARR $1.03,95 \%$ CI 1.02-1.05) prescription fill rates, compared to men.

These findings may indicate poorer disease control, greater asthma severity and poorer access to specialist asthma care in women.

@ERSpublications

Older women with asthma have lower rates of spirometry, asthma specialist visits and asthma controller fill rates, and higher rates of ED visits for asthma, physician office visits for asthma and asthma reliever fill rates, compared to men http://bit.ly/33PfMD6

Cite this article as: To T, Gray N, Ryckman K, et al. Sex differences in health services and medication use among older adults with asthma. ERJ Open Res 2019; 5: 00242-2019 [https://doi.org/ $10.1183 / 23120541.00242-2019]$.

This article has supplementary material available from openres.ersjournals.com

Received: 9 Sept 2019 | Accepted after revision: 17 Oct 2019

Copyright $\odot$ ERS 2019. This article is open access and distributed under the terms of the Creative Commons Attribution Non-Commercial Licence 4.0. 


\section{Introduction}

Research has demonstrated that sex is an independent predictor of disease among the chronic disease populations with cardiovascular disease and diabetes [1-6]. However, population-based research on sex differences is lacking for respiratory conditions, and in particular for the older subpopulation of people with asthma.

Asthma affects approximately 334 million individuals worldwide [7], with the incidence and prevalence varying by age and sex [8-10]. The burden of asthma is particularly high at age 5-19 years and >60 years, with the former contributing the most to disability and the latter to premature deaths [7].

Sex differences in asthma prevalence, health services use (HSU), morbidity and mortality have been well reported in the literature; however, many studies excluded the older adult population [8, 10]. ScHATz and CAMARGO JR [10] studied asthma prevalence, healthcare utilisation and medications in those with asthma aged 2-64 years using data from Southern California Kaiser-Permanente Medical Care Program (San Diego, CA, USA). The authors found higher asthma healthcare utilisation in males during childhood and teenage years, but higher utilisation for females among those aged 23-64 years. While girls typically have a lower risk of asthma than boys, women become more susceptible to developing asthma after puberty $[8,10,11]$.

Recent research studies in Australia [12] and the USA [13] have shown that asthma is more severe in older women compared to men. Compared to men, older women (aged $\geqslant 50$ years) with asthma tend to have more frequent asthma acute HSU [13], a doubled risk of emergency department (ED) visits for asthma [14] and a 1.2-fold increased risk of death, independent of age, comorbidities, demographics, residence and social support [12].

In Canada, the prevalence of asthma is higher in older women than in older men. Among Canadians aged $\geqslant 55$ years, asthma is prevalent in about $9 \%$ of women compared to 6-7\% of men [15]. Similarly, in Ontario, the asthma prevalence is $60 \%$ and $33 \%$ higher in women than in men for those aged 50-69 years and $>70$ years, respectively [16]

Despite the growing body of literature on sex differences among older individuals with asthma, few studies have used population-based data. Thus, the objective of this study was to use population-based data from Ontario, Canada, to compare rates of asthma-specific health services and medication use, between older men and women with asthma. Our findings will better the understanding of sex-specific gaps of asthma burden.

\section{Methods}

Study design and data sources

This population-based study used a cohort design with data acquired from Ontario health administrative databases housed at ICES (formerly the Institute for Clinical Evaluative Sciences) in Toronto, Ontario, Canada. In Ontario, there is routine collection of HSU data for research purposes. Using health administrative data allowed us to study HSU over time without selection or response bias. The Ontario Health Insurance Plan (OHIP) claims database captures physician office visits, the National Ambulatory Care Reporting System (NACRS) captures ED visits, the Canadian Institute for Health Information Discharge Abstract Database (CIHI-DAD) captures hospitalisations, the Ontario Drug Benefit (ODB) database captures prescriptions filled, and the provincial Registered Persons Database (RPDB) provides sociodemographic information.

\section{Study population}

The study cohort included 209054 Ontario residents aged $\geqslant 66$ years with prevalent asthma on 1 April 2010 (which served as baseline). Asthma prevalence was defined using the Ontario Asthma Surveillance Information System (OASIS), which identifies individuals with asthma based on a validated health administrative definition of two or more outpatient visits for asthma in two consecutive years, or one hospitalisation for asthma. This definition has demonstrated a sensitivity of $84 \%$ and specificity of $76 \%$ in the Ontario adult population [17]. Individuals were excluded from the cohort if they had missing data for age or Ontario residence postal code, or if they did not have a valid Ontario health card number (i.e. were uninsured). Participants were followed from baseline until 31 March 2016, death, or they moved outside of Ontario.

\section{Exposure and outcome measurement}

The primary exposure was sex, acquired from the RPDB. The primary outcomes were rates of asthma-specific spirometry, ED, hospitalisation, physician office and specialist visits, and prescription fill rates for asthma controller and reliever medications. Rates were derived from outcome counts divided by total follow-up time for each individual. Visit types were identified using International Classification of Disease, 10th Revision (ICD-10) codes and OHIP billing codes. Asthma was defined using ICD-10 codes J45 and J46, and OHIP code 493. 


\section{Covariates}

Socioeconomic status was measured by proxy using the 2011 Ontario Marginalization Index [18]. The Ontario Marginalization Index uses census data to measure four dimensions of marginalisation (i.e. material deprivation, dependency, ethnic concentration and residential instability) at the neighbourhood level. Individuals are assigned a value from 1 (least marginalised) to 5 (most marginalised) for each domain based on their residence location at baseline. Income was also measured by proxy using income quintile ( 1 to 5 ), with individuals residing in census divisions with the lowest $20 \%$ household income assigned 1, and those in the highest $20 \%$ assigned 5. Residence at baseline was measured as "rural" if the individual resided in a community of $\leqslant 10000$ people, and otherwise as "urban" [19]. Residence at baseline was also measured by local health integration networks (LHINs). LHINs are geographical regions established across Ontario to plan, fund and coordinate local community-based health services [19]. A co-diagnosis of COPD comorbidity was adjusted as a covariate. COPD was defined using a validated health administrative definition of one or more ambulatory care claims for COPD in two consecutive years, or one hospitalisation for COPD [20]. In addition, the number of comorbidities was defined using the Collapsed Aggregated Diagnosis Groups (CADGs) from the Johns Hopkins ACG System Version 10. The CADGs categorise International Classification of Diseases, 9th Revision (ICD-9) and ICD-10 diagnostic codes based on severity and the likelihood of persistence of the respective health condition [21]. The CADGs consist of a series of 12 variables that measure the expected healthcare utilisation of an individual in the 2 years prior to a time point. Other covariates included age at baseline and age at asthma prevalence.

\section{Statistical analysis}

Baseline characteristics were described for men and women using frequencies and proportions for categorical variables and means with standard deviations for continuous variables. Differences between men and women on baseline characteristics were compared using a standardised difference score, in order to provide a statistical measure of differences between groups that would not be influenced by the study's large sample size. Crude yearly rates of HSU and prescriptions filled were calculated and compared between men and women. The majority of asthma controller prescriptions filled were for a 1-month supply, while the majority of asthma reliever prescriptions filled were for $\leqslant 1$ month. Multivariate negative binomial regression was used to measure the association between sex and health services and medication outcomes, while adjusting for important confounding variables as outlined. Follow-up time was included as an offset. A rate ratio with $95 \%$ confidence interval was produced. In all analyses, a p-value of $<0.05$ was used to determine statistical significance. All analyses were carried out using SAS Enterprise guide 6.1 (SAS Institute Inc., Cary, NC, USA).

\section{Research ethics}

Ethics approval for this study was obtained from the Hospital for Sick Children, in Toronto. Individual participant consent was not required for this study due to exclusive use of large, anonymised population databases.

\section{Results}

\section{Descriptive statistics}

The study cohort consisted of 209054 individuals aged $\geqslant 66$ years with prevalent asthma who resided in Ontario, of which 131628 (63\%) were women (table 1). Approximately half of the cohort were aged 66-75 years (younger age category) at baseline (1 April 2010) (52\% and 54\%, for women and men, respectively). The average asthma duration (time from registered asthma onset to study entry) was 12 years for women and 11 years for men. Women and men were both followed for an average of 5 years from baseline to the end of the study (31 March 2016).

Women and men were similar with regard to income quintile and across several dimensions of the Ontario Marginalization Index. However, a greater proportion of women (31\%) compared to men (24\%) were in the highest quintile of the residential instability dimension, which provides a measure of the proportion of the population that lives alone/in apartment buildings/in non-owned properties, among others. A similar proportion of women (11\%) and men (13\%) lived in rural areas, while the majority of the population resided in urban locations at baseline.

A lower proportion of women had histories of COPD (57\%), diabetes (37\%), acute myocardial infarction (7\%) and non-lung, non-breast cancer (14\%) compared to men (66\%, 42\%, 13\% and 28\%, respectively). However, the overall burden of comorbidity, measured through the number of CADGs, was similar between women and men (5.6 \pm 2.1 and 5.7 \pm 2.1$)$. Approximately one-fifth of women $(21 \%)$ died during follow-up, compared to one-quarter of men (25\%). For both women and men, the most common causes of death were diseases of the circulatory system, neoplasms and diseases of the respiratory system. 


\section{TABLE 1 Characteristics of the study population ${ }^{\#}$ by sex}

\begin{tabular}{|c|c|c|c|}
\hline Characteristic & Women & Men & Standardised difference ${ }^{\pi}$ \\
\hline Subjects & 131628 & 77426 & \\
\hline \multicolumn{4}{|c|}{ Age group at asthma prevalence years } \\
\hline $47-59$ & 43245 (32.85) & 24712 (31.92) & 0.020 \\
\hline $60-69$ & 52023 (39.52) & $32297(41.71)$ & 0.045 \\
\hline $70-99$ & $36360(27.62)$ & 20417 (26.37) & 0.028 \\
\hline \multicolumn{4}{|l|}{ Age group at baseline ${ }^{+}$years } \\
\hline $66-75$ & $68022(51.68)$ & 41609 (53.74) & 0.041 \\
\hline $76-85$ & 46769 (35.53) & $28384(36.66)$ & 0.024 \\
\hline $86-99$ & 16837 (12.79) & $7433(9.60)$ & 0.100 \\
\hline Asthma duration years & $11.88 \pm 5.43$ & $11.41 \pm 5.62$ & 0.086 \\
\hline Follow-up time years & $5.20 \pm 1.60$ & $5.04 \pm 1.73$ & 0.097 \\
\hline \multicolumn{4}{|l|}{ Income quintile } \\
\hline 1 (lowest) & 28790 (21.87) & 14771 (19.08) & 0.069 \\
\hline 2 & $28197(21.42)$ & $16074(20.76)$ & 0.016 \\
\hline 3 & 25904 (19.68) & 15527 (20.05) & 0.009 \\
\hline 4 & 24845 (18.88) & $15550(20.08)$ & 0.031 \\
\hline 5 (highest) & 23385 (17.77) & 15227 (19.67) & 0.049 \\
\hline Missing & 507 (0.39) & $277(0.36)$ & 0.005 \\
\hline \multicolumn{4}{|l|}{ Rural } \\
\hline Yes & 15065 (11.45) & 9951 (12.85) & 0.043 \\
\hline No & 116531 (88.53) & 67455 (87.12) & 0.043 \\
\hline Missing & $32(0.02)$ & 20 (0.03) & 0.001 \\
\hline \multicolumn{4}{|l|}{ Ontario Marginalization Index } \\
\hline \multicolumn{4}{|l|}{ Deprivation quintile } \\
\hline 1 (lowest) & 24023 (18.25) & 15166 (19.59) & 0.034 \\
\hline 2 & $24304(18.46)$ & $15019(19.40)$ & 0.024 \\
\hline 3 & $25673(19.50)$ & 15392 (19.88) & 0.009 \\
\hline 4 & $26892(20.43)$ & $15418(19.91)$ & 0.013 \\
\hline 5 (highest) & 28941 (21.99) & 15523 (20.05) & 0.048 \\
\hline Missing & $1795(1.36)$ & 908 (1.17) & 0.017 \\
\hline \multicolumn{4}{|l|}{ Dependency quintile } \\
\hline 1 (lowest) & $16502(12.54)$ & 10472 (13.53) & 0.029 \\
\hline 2 & $20221(15.36)$ & 13107 (16.93) & 0.043 \\
\hline 3 & $22224(16.88)$ & 13891 (17.94) & 0.028 \\
\hline 4 & 25518 (19.39) & $15609(20.16)$ & 0.019 \\
\hline 5 (highest) & $45368(34.47)$ & 23439 (30.27) & 0.090 \\
\hline Missing & $1795(1.36)$ & $908(1.17)$ & 0.017 \\
\hline \multicolumn{4}{|l|}{ Ethnic concentration quintile } \\
\hline 1 (lowest) & $26616(20.22)$ & $15876(20.5)$ & 0.007 \\
\hline 2 & 23409 (17.78) & 13928 (17.99) & 0.005 \\
\hline 3 & 23987 (18.22) & $13710(17.71)$ & 0.013 \\
\hline 4 & $25292(19.21)$ & $14510(18.74)$ & 0.012 \\
\hline 5 (highest) & 30529 (23.19) & 18494 (23.89) & 0.016 \\
\hline Missing & $1795(1.36)$ & 908 (1.17) & 0.017 \\
\hline \multicolumn{4}{|l|}{ Residential instability quintile } \\
\hline 1 (lowest) & 17104 (12.99) & 12339 (15.94) & 0.084 \\
\hline 2 & 20979 (15.94) & 14481 (18.70) & 0.073 \\
\hline 3 & 24155 (18.35) & 15385 (19.87) & 0.039 \\
\hline 4 & $27165(20.64)$ & $15550(20.08)$ & 0.014 \\
\hline 5 (highest) & $40430(30.72)$ & $18763(24.23)$ & 0.150 \\
\hline Missing & 1795 (1.36) & $908(1.17)$ & 0.017 \\
\hline \multicolumn{4}{|c|}{ Prevalence of other health conditions } \\
\hline COPD & $75507(57.36)$ & 50895 (65.73) & 0.170 \\
\hline Diabetes & $49022(37.24)$ & $32845(42.42)$ & 0.110 \\
\hline Hypertension & $112497(85.47)$ & 64136 (82.84) & 0.072 \\
\hline Stroke & $39887(30.3)$ & 24054 (31.07) & 0.017 \\
\hline Congestive heart failure & 34517 (26.22) & $22578(29.16)$ & 0.066 \\
\hline Acute myocardial infarction & 9529 (7.24) & 9899 (12.79) & 0.190 \\
\hline Lung cancer & 4272 (3.25) & 3315 (4.28) & 0.055 \\
\hline Breast cancer & 10435 (7.93) & $157(0.2)$ & 0.400 \\
\hline
\end{tabular}




\begin{tabular}{|c|c|c|c|}
\hline Characteristic & Women & Men & Standardised difference ${ }^{\pi}$ \\
\hline Other cancer & $17814(13.53)$ & $21560(27.85)$ & 0.360 \\
\hline Number of CADGs & $5.62 \pm 2.07$ & $5.74 \pm 2.07$ & 0.056 \\
\hline \multicolumn{4}{|l|}{ Died during follow-up } \\
\hline Yes & $27487(20.88)$ & 19254 (24.87) & 0.095 \\
\hline No & $104141(79.12)$ & $58172(75.13)$ & 0.095 \\
\hline \multicolumn{4}{|l|}{ Cause of death } \\
\hline Diseases of the circulatory system & 7778 (28.3) & 5406 (28.08) & 0.005 \\
\hline Neoplasms & $5938(21.6)$ & 4849 (25.18) & 0.085 \\
\hline Diseases of the respiratory system & $5125(18.65)$ & $3882(20.16)$ & 0.038 \\
\hline Mental and behavioural disorders & $1888(6.87)$ & $881(4.58)$ & 0.099 \\
\hline Diseases of the nervous system & 996 (3.62) & $578(3.00)$ & 0.035 \\
\hline Other & 5490 (19.97) & 3455 (17.94) & 0.052 \\
\hline Missing & $272(0.99)$ & 203 (1.05) & 0.006 \\
\hline
\end{tabular}

Rates of asthma-specific health services use and prescriptions by sex

Women had fewer spirometry visits $(24.1 \pm 64.6$ per 100 person-years $)$ and physician office visits $(30.4 \pm 97.8$ per 100 person-years) compared to men (32.4 991.2 and $31.7 \pm 107.9$ per 100 person-years, respectively) (table 2). Rates of asthma-specific ED visits and hospitalisations were slightly higher among women $(0.9 \pm 12.1$ and $0.8 \pm 20.5$ per 100 person-years, respectively) compared to men $(0.7 \pm 6.8$ and $0.4 \pm 9.7$ per 100 person-years, respectively). Rates of asthma-specific specialist visits were similar between women and men (about $10.3 \pm 45.0$ visits per 100 person-years for both sexes). Women filled fewer asthma controller (3.5 \pm 5.1 per person-year) and reliever $(1.8 \pm 3.8$ per person-year) prescriptions compared to men $(4.0 \pm 6.0$ and $2.0 \pm 4.8$ per person-year, respectively).

Adjusted relative rates of asthma-specific health services use and prescriptions by sex

The results of the multivariable negative binomial regression revealed that women had a $13 \%$ lower rate of spirometry (adjusted relative rate (ARR) $0.87,95 \%$ CI $0.85-0.89$ ) and a $43 \%$ higher rate of ED visits for asthma (ARR 1.43, 95\% CI 1.33-1.53) (figure 1). Women also had a higher rate of physician office visits for asthma (ARR 1.03, 95\% CI 1.01-1.05) and a lower rate of specialist visits for asthma (ARR 0.93, 95\% CI $0.90-0.96$ ). The negative binomial regression model for hospitalisations did not converge (logistic regression results are presented in the online supplementary material). With regard to medication use, women had a lower fill rate of asthma controller prescriptions (ARR 0.98, 95\% CI 0.97-0.99) and a higher fill rate of asthma reliever prescriptions (ARR 1.03, 95\% CI 1.02-1.05) compared to men. The multivariate regression models were adjusted for age at baseline, age at asthma prevalence, CADGs, Ontario

TABLE 2 Mean rates of asthma-specific health services use (HSU) and prescriptions by sex

\begin{tabular}{lccc} 
Outcome & Women & Men & Standardised difference $^{\#}$ \\
\hline Subjects & 131628 & 77426 & \\
Asthma-specific HSU per 100 person-years & & & \\
$\quad$ Spirometry visits & $24.1 \pm 64.6$ & $32.4 \pm 91.2$ & $\mathbf{0 . 1 0 5}$ \\
$\quad$ Asthma-specific ED visits & $0.9 \pm 12.1$ & $0.7 \pm 6.8$ & 0.025 \\
Asthma-specific hospitalisations & $0.8 \pm 20.5$ & $0.4 \pm 9.7$ & 0.029 \\
$\quad$ Asthma-specific physician office visits & $30.4 \pm 97.8$ & $31.7 \pm 107.9$ & 0.012 \\
$\quad$ Asthma-specific specialist visits & $10.3 \pm 45.0$ & $10.4 \pm 47.2$ & 0.003 \\
Asthma-specific medication use per person-year & & & \\
Asthma controller prescriptions & $3.5 \pm 5.1$ & $4.0 \pm 6.0$ & 0.088 \\
Asthma reliever prescriptions & $1.8 \pm 3.8$ & $2.0 \pm 4.8$ & 0.046
\end{tabular}

Data are presented as $\mathrm{n}$ or mean $\pm \mathrm{SD}$, unless otherwise stated. ${ }^{\#}$ : standardised difference of $>0.1$ generally considered different (shown in bold). 


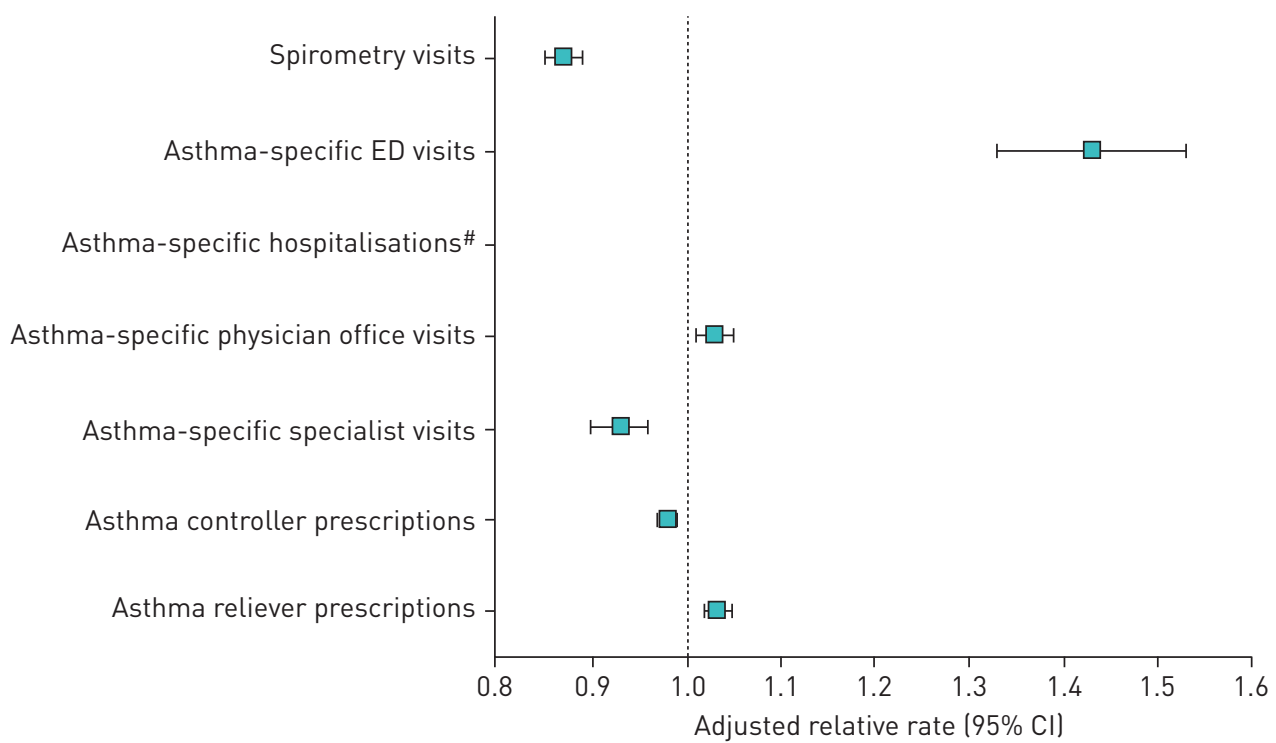

FIGURE 1 Forest plot for adjusted relative rates of asthma-specific health services use and prescriptions for women versus men. The multivariate negative binomial regression models were adjusted for age at baseline, age at asthma prevalence, Collapsed Aggregated Diagnosis Groups, Ontario Marginalization Index, local health integration networks, rurality and COPD; offset by loglyears observed). Data are shown for the study population ( $n=209054)$. ED: emergency department. \#: the negative binomial regression model did not converge.

Marginalization Index, LHINs, rurality and COPD. The unadjusted and adjusted relative rates are presented in the online supplementary material.

\section{Discussion}

Recent research studies have suggested that asthma is more severe in older women compared to men [12-14]. However, the extent of this difference remains poorly understood. Using health administrative data from Ontario, our study was one of the largest population-based cohort studies to examine sex differences in older adults with asthma. In this study, we found that, compared to men, older women with asthma had lower rates of spirometry and specialist visits for asthma, and higher rates of ED and physician office visits for asthma. We also found that women had a lower asthma controller prescription fill rate and a higher asthma reliever prescription fill rate, compared to men. These differences may suggest poorer disease control, higher asthma severity and poorer access to specialist asthma care in older women. These results are consistent with the limited published literature.

Previous research has suggested that, while men and women may perceive asthma symptoms similarly, their approach to managing asthma symptoms and healthcare-seeking behaviour may differ [10]. Women tend to have an altered perception of breathlessness [14]. EBIHARA et al. [22] found that older individuals (64\% female) had a decreased perception of airflow obstruction and this was associated with greater medication costs, hospitalisations and mortality, compared to those with a better perception of dyspnoea. To overcome the decreased perception of breathlessness, the National Institutes of Health (NIH) asthma guidelines recommend periodic assessment of asthma control with spirometry [14, 23]. However, similar to our observation, SIKJÆR et al. [24] found that significantly fewer women than men had undergone pulmonary function testing, based on an Internet-based questionnaire administered to a random sample of the Danish population. The lower use of spirometry to measure and monitor lung function may be due to either provider or patient behaviours. Therefore, it is important to increase awareness of the importance of monitoring asthma control, such as with spirometry, among both providers and individuals with asthma, to ensure patients receive the best asthma care.

Researchers in the USA have found that asthma specialists provide more thorough care than primary care physicians [25]. Individuals treated by an asthma specialist were more likely to own and use a steroid inhaler and peak flow meter daily [25]. Asthma specialists also provided more information on preventing and controlling asthma attacks [25]. However, we found that women had lower rates of specialist visits for asthma compared to men. Among the cardiovascular disease population, a lower referral rate to cardiac rehabilitation has been widely reported among women [26]. Our findings may indicate a similar lower referral rate to specialist asthma care in older women with asthma. Improving access to asthma specialist 
care among older women with asthma may lead to improved asthma disease management and reduced asthma morbidity.

In this study, higher rates of ED and physician office visits for asthma were observed among older women, compared to men. ANTONicelli et al. [27] found that HSU was positively associated with asthma severity. Therefore, these findings may indicate greater asthma severity among women. The trend of greater asthma morbidity in older women is also observed in other developed countries such as Australia and the USA $[12,14]$. Researchers have suggested that there are multiple factors at play: biological, lifestyle, societal, environmental, diagnostic, disease management, comorbidities (e.g. COPD) and access to care. The extra burden may be due to sex-specific differences in biological and physiological features, such as greater fluctuations in hormone levels [28, 29], generally lower IgE levels [30], greater prevalence of atopy [28], smaller lung size and airway calibre $[8,31]$ and thus expedited declines in airway capacity [28] in older women. Increased asthma HSU in older women may be related to the menopause, which increases the risk of asthma attacks [32, 33]. It may also be related to poor asthma control [28], such as improper use of respiratory inhalers [34] and a lower asthma controller prescription rate [11,35].

We found that women had a lower asthma controller prescription fill rate and a higher asthma reliever prescription fill rate, compared to men. In a national population-based study in South Korea, similar to our observation, CHOI et al. [35] found that the inhaled corticosteroid prescription rate among individuals with asthma was lower among females and those aged $>90$ years. Underutilisation of controller medications among older adults with asthma may lead to preventable HSU [36]. Additionally, since asthma reliever medications are used to rapidly treat asthma symptoms, a higher asthma reliever prescription fill rate among older women may indicate more poorly controlled asthma [36].

The descriptive mean rates showed no difference in the outcomes between women and men except for spirometry visit rates. However, when adjusted for the confounders, all rates indicated statistically significant differences. Specifically, women had a statistically higher relative rate for ED visits, physician office visits and reliever medications filled, but lower relative rate for spirometry visits, specialist visits and controller medications filled. This suggested that negative confounding influenced the unadjusted observed rates.

Strengths of this study include the use of health administrative data to collect information about asthma-specific HSU and prescriptions in Ontario. This data source allowed us to study longitudinal HSU without selection or response bias, and may allow us to generalise our results to other populations of older individuals with asthma. However, using health administrative data, we were limited in our ability to adjust for important lifestyle factors that could confound the relationship between men and women and HSU, such as smoking, body mass index and health-seeking behaviours. Using this data source, we were also limited in our ability to examine prescriptions written and prescriptions taken. Furthermore, our ability to identify individuals with asthma was limited by the sensitivity and specificity of our asthma definition. It is possible that individuals in our study cohort were misclassified as individuals with asthma.

Our findings may help inform the future development of prevention and management initiatives that aim to decrease the risk of poor health outcomes and thereby improve the quality of life in older women living with asthma. A better understanding about the sex-specific risk factors for asthma morbidity in older women, particularly the high-utilisation group, would also facilitate targeted monitoring in the future.

\section{Conclusion}

Older women with asthma had lower rates of spirometry, asthma specialist visits and asthma controller fill rates, and higher rates of ED visits for asthma, physician office visits for asthma and asthma reliever fill rates, compared to men. These differences may indicate poorer disease control, greater asthma severity and poorer access to specialist asthma care in women. Future research should aim to identify factors that explain these sex differences, to ensure optimal asthma care for all.

Conflict of interest: None declared.

Support statement: This study was funded by the Ontario Ministry of Health and Long-Term Care (MOHLTC), grant number HLTC3968IT-2018-501. Data were provided by ICES (formerly the Institute for Clinical Evaluative Sciences), which is funded by an annual grant from the MOHLTC. The analyses, conclusions, opinions and statements expressed herein are solely those of the authors and do not reflect those of the funding or data sources; no endorsement is intended or should be inferred. Funding information for this article has been deposited with the Crossref Funder Registry. 


\section{References}

1 Brännström J, Hamberg K, Molander L, et al. Gender disparities in the pharmacological treatment of cardiovascular disease and diabetes mellitus in the very old: an epidemiological, cross-sectional survey. Drugs Aging 2011; 28: 993-1005.

2 Short SE, Yang YC, Jenkins TM. Sex, gender, genetics, and health. Am J Public Health 2013; 103: Suppl. 1, S93-S101.

3 Hussain MA, Lindsay TF, Mamdani M, et al. Sex differences in the outcomes of peripheral arterial disease: a population-based cohort study. CMAJ Open 2016; 4: E124-E131.

4 Dhamoon MS, Liang JW, Zhou L, et al. Sex differences in outcomes after stroke in patients with diabetes in Ontario, Canada. J Stroke Cerebrovasc Dis 2018; 27: 210-220.

5 Kapral MK, Degani N, Hall R, et al. Gender differences in stroke care and outcomes in Ontario. Womens Health Issues $2011 ; 21: 171-176$.

6 Krämer HU, Rüter G, Schöttker B, et al. Gender differences in healthcare utilization of patients with diabetes Am J Manag Care 2012; 18: 362-369.

7 Global Asthma Network. The Global Asthma Report 2014. Available from: http://globalasthmareport.org/2014/

8 de Marco R, Locatelli F, Sunyer J, et al. Differences in incidence of reported asthma related to age in men and women. A retrospective analysis of the data of the European Respiratory Health Survey. Am J Respir Crit Care Med 2000; 162: 68-74

9 Chen Y, Stewart P, Johansen H, et al. Sex difference in hospitalization due to asthma in relation to age. J Clin Epidemiol 2003; 56: 180-187.

10 Schatz M, Camargo CA Jr. The relationship of sex to asthma prevalence, health care utilization, and medications in a large managed care organization. Ann Allergy Asthma Immunol 2003; 91: 553-558.

11 Bloom CI, Nissen F, Douglas IJ, et al. Exacerbation risk and characterisation of the UK's asthma population from infants to old age. Thorax 2018; 73: 313-320.

12 Eftekhari P, Forder PM, Majeed T, et al. Impact of asthma on mortality in older women: an Australian cohort study of 10,413 women. Respir Med 2016; 119: 102-108.

13 Moorman JE, Akinbami LJ, Bailey CM, et al. National surveillance of asthma: United States, 2001-2010. Vital Health Stat 2012: 3: 1-58.

14 Baptist AP, Hamad A, Patel MR. Special challenges in treatment and self-management of older women with asthma. Ann Allergy Asthma Immunol 2014; 113: 125-130.

15 Statistics Canada. Asthma, 2014. Date last updated: 27 November 2015. Date last accessed: 13 November 2017 www150.statcan.gc.ca/n1/pub/82-625-x/2015001/article/14179-eng.htm

16 Gershon AS, Guan J, Wang C, et al. Trends in asthma prevalence and incidence in Ontario, Canada, 1996-2005: a population study. Am J Epidemiol 2010; 172: 728-736.

17 Gershon AS, Wang C, Guan J, et al. Identifying patients with physician-diagnosed asthma in health administrative databases. Can Respir J 2009; 16: 183-188.

18 Matheson FI, Ontario Agency for Health Protection and Promotion (Public Health Ontario). 2011 Ontario Marginalization Index: User guide. Toronto, St. Michael's Hospital, 2017. Available from: https://www.publichealth ontario.ca/-/media/documents/on-marg-user-2011.pdf?la=en

19 Ontario Ministry of Health and Long-Term Care. Health Analyst's Toolkit. Toronto, Health Analytics Branch, 2012

20 Gershon AS, Wang C, Guan J, et al. Identifying individuals with physician diagnosed COPD in health administrative databases. COPD 2009; 6: 388-394.

21 The Johns Hopkins University. The Johns Hopkins ACG System. https://hopkinsacg.org/?option=com content\&view=article\&id=55\%3Adescribing-morbidity-burden\&catid=37\%3Asystem-components \&Itemid=315

22 Ebihara S, Niu K, Ebihara T, et al. Impact of blunted perception of dyspnea on medical care use and expenditure, and mortality in elderly people. Front Physiol 2012; 3: 238.

23 National Institutes of Health. Expert Panel Report 3: Guidelines for the Diagnosis and Management of Asthma Bethesda, National Heart, Lung, and Blood Institute, 2007.

24 Sikjær MG, Hilberg O, Fløe A, et al. Lack of awareness towards smoking-related health risks, symptoms related to COPD, and attitudinal factors concerning smoking: an Internet-based survey conducted in a random sample of the Danish general population. Eur Clin Respir J 2018; 5: 1506235.

25 Legorreta AP, Christian-Herman J, O'Connor RD, et al. Compliance with national asthma management guidelines and specialty care: a health maintenance organization experience. Arch Intern Med 1998; 158: 457-464.

26 Colella TJ, Gravely S, Marzolini S, et al. Sex bias in referral of women to outpatient cardiac rehabilitation? A meta-analysis. Eur J Prev Cardiol 2015; 22: 423-441.

27 Antonicelli L, Bucca C, Neri M, et al. Asthma severity and medical resource utilisation. Eur Respir J 2004; 23: 723-729.

28 Redline S, Gold D. Challenges in interpreting gender differences in asthma. Am J Respir Crit Care Med 1994; 150: 1219-1221.

29 Caracta CF. Gender differences in pulmonary disease. Mt Sinai J Med 2003; 70: 215-224.

30 Wüthrich B, Schindler C, Medici TC, et al. IgE levels, atopy markers and hay fever in relation to age, sex and smoking status in a normal adult Swiss population. SAPALDIA (Swiss Study on Air Pollution and Lung Diseases in Adults) Team. Int Arch Allergy Immunol 1996; 111: 396-402.

31 Brooks LJ, Byard PJ, Helms RC, et al. Relationship between lung volume and tracheal area as assessed by acoustic reflection. J Appl Physiol 1988; 64: 1050-1054.

32 Balzano G, Fuschillo S, Melillo G, et al. Asthma and sex hormones. Allergy 2001; 56: 13-20.

33 Romieu I, Fabre A, Fournier A, et al. Postmenopausal hormone therapy and asthma onset in the E3N cohort. Thorax 2010; 65: 292-297.

34 Goodman DE, Israel E, Rosenberg M, et al. The influence of age, diagnosis, and gender on proper use of metered-dose inhalers. Am J Respir Crit Care Med 1994; 150: 1256-1261.

35 Choi JY, Yoon HK, Lee JH, et al. Nationwide use of inhaled corticosteroids by South Korean asthma patients: an examination of the Health Insurance Review and Service database. I Thorac Dis 2018; 10: 5405-5413.

36 Hartert TV, Togias A, Mellen BG, et al. Underutilization of controller and rescue medications among older adults with asthma requiring hospital care. J Am Geriatr Soc 2000; 48: 651-657. 